\title{
Bedaquiline: A Mini Review
}

\author{
Nandhini Saravanabavan ${ }^{1}$, Padmavathi Shanmuganathan ${ }^{2}$, Manimekalai Kumarappan $^{3}$
}

\begin{abstract}
Tuberculosis is a pandemic, chronic infectious disease which affects more than 10 million people in the world. About $3.5 \%$ of patients of newly diagnosed have reported having multidrug-resistant tuberculosis (MDR-TB). The standard treatment of MDR-TB has many cons like high cost, less effectiveness, and more drug interactions. Thus, to overcome these disadvantages a new novel compound which belongs to the diarylquinolone group called bedaquiline has been developed as a part of combination therapy in adults with MDR-TB for its bactericidal activity. It mainly acts by inhibiting the adenosine triphosphate synthase enzyme of Mycobacterium tuberculosis. This drug was introduced as a new addition to the standard TB regimen after 50 years.
\end{abstract}

Keywords: Antitubercular drug, Bedaquiline, Multidrug-resistant tuberculosis.

Annals of SBV (2019): 10.5005/jp-journals-10085-8101

Tuberculosis is a chronic infectious disease caused by Mycobacterium tuberculosis associated with significant morbidity and mortality. ${ }^{1,2}$ According to World Health Organization (WHO), it affects more than 10 million people in the world and labeled as a worldwide pandemic. ${ }^{3}$ It is considered as the second most leading cause of death after HIV. ${ }^{4}$ The treatment outcome for drug-sensitive TB is highly effective when compared with the treatment outcome of MDR-TB. About $3.5 \%$ of patients of newly diagnosed have MDR-TB. ${ }^{5}$

Multidrug-resistant tuberculosis is defined as those patients who shows resistance to the most important two first-line drugs that are isoniazid and rifampicin. ${ }^{6}$ There are many problems reported in the treatment of MDR regimen, such as high cost, less effectiveness, with more drug interactions, and longer onset of action. ${ }^{7}$ Thus, to overcome these drawbacks a new novel compound has been developed called bedaquiline. After 50 years, ${ }^{8}$ the new antitubercular drug with a different mechanism of action was approved by Food and Drug Administration on December 28, 2012 as a part of combination therapy in adults with MDR-TB for its bactericidal activity over M. tuberculosis. ${ }^{9}$ Mycobacterium tuberculosis requires mycobacterial adenosine triphosphate (ATP) synthase to synthesis ATP. Bedaquiline which belongs to diarylquinoline group ${ }^{10}$ inhibits the enzyme mycobacterial ATP synthase and prevents the ATP synthesis in bacteria leading to bacterial death. Bedaquiline specifically binds to subunit $C$ of the mycobacterial ATP synthase enzyme. The gene which encodes the $C$ subunit of the enzyme is atpE. ${ }^{11}$ Any mutation in this gene can alter the effect of bedaquiline.

Some of the preclinical studies were also conducted in guinea pigs in which bedaquiline was administered for 6 weeks and found to be effective in the eradication of $M$. tuberculosis from both primary and secondary lesions in lung granuloma. ${ }^{12}$ In mice, the combination of bedaquiline-rifapentine-pyrazinamide produces significant bactericidal activity. ${ }^{13}$ There was a synergistic interaction between pyrazinamide and bedaquiline ${ }^{14}$ as pyrazinamide being indirect an inhibitor of ATP synthase. ${ }^{15}$

In the first-stage phase 2, randomized, controlled trial which were conducted in 47 patients to evaluate whether addition of bedaquiline to the standard second-line antituberculosis regimen would alter the conversion of sputum culture to negative showed that the 23 patients who took (TMC207) bedaquiline $400 \mathrm{mg}$ daily for 2 weeks followed by 200 mg three times a week for 6 weeks had

\begin{abstract}
${ }^{1-3}$ Department of Pharmacology, Mahatma Gandhi Medical College and Research Institute, SBV Deemed to be University, Puducherry,
\end{abstract} India

Corresponding Author: Nandhini Saravanabavan, Department of Pharmacology, Mahatma Gandhi Medical College and Research Institute, SBV Deemed to be University, Puducherry, India, Phone: +91 9443534537, e-mail: nandhinisaravanabavan@gmail.com

How to cite this article: Saravanabavan N, Shanmuganathan $P$, Kumarappan M. Bedaquiline: A Mini Review. Ann SBV 2019;8(1):2-4.

Source of support: Nil

Conflict of interest: None

required only less time for sputum conversion to negative when compared with the patients who took placebo. Most frequently patients experienced nausea with bedaquiline intake and QT prolongation also occurred in the patients taking bedaquiline compared with the placebo. ${ }^{16}$

In the second stage of phase $2 \mathrm{~b}$, randomized, double-blind, multicenter placebo-controlled trial involved 79 patients who took bedaquiline had shown the reduction in median time to conversion of culture from positive to negative when compared with placebo from 125 days to 83 days. Most frequently patient experienced nausea, vomiting, and arthralgia while taking bedaquiline. The most serious adverse effect observed is QT prolongation which was markedly higher in patients taking bedaquiline when compared with placebo. When the bedaquiline treatment was stopped after 24 weeks, the improvement in QT prolongation was observed. Number of death is also more in patients taking bedaquiline when compared with placebo. The total number of death bedaquiline groups is 10 and 2 in the placebo group. Out of 10 death in bedaquiline group, 5 occurred due to progression of the disease, 4 occurred due to unrelated cause, and 1 occurred due to motor vehicle accident. ${ }^{17}$

A retrospective cohort study for 6 months was conducted to determine the tolerability and short-term microbiological efficacy. After 6 months of bedaquiline initiation culture conversion was achieved in $97 \%$ with 85 days requirement for median time to culture conversion. But the most important adverse effect observed was an elevation in liver enzymes and QT prolongation. One death also occurred during the study period but it was reported as unrelated to antituberculosis treatment. ${ }^{18}$

(อ) The Author(s). 2019 Open Access This article is distributed under the terms of the Creative Commons Attribution 4.0 International License (https://creativecommons. org/licenses/by-nc/4.0/), which permits unrestricted use, distribution, and non-commercial reproduction in any medium, provided you give appropriate credit to the original author(s) and the source, provide a link to the Creative Commons license, and indicate if changes were made. The Creative Commons Public Domain Dedication waiver (http://creativecommons.org/publicdomain/zero/1.0/) applies to the data made available in this article, unless otherwise stated. 
An interim cohort analysis was conducted in South Africa where a load of extensive drug-resistant tuberculosis (XDR) is higher. Out of 63,48 patients with 6 months follow-up either remained culture-negative or achieved culture conversion after bedaquiline initiation. Overall, the study shows that bedaquiline may be safe and also efficacious even in those patients with HIV infection on antiretroviral therapy and also in patients with pre-XDR and XDR. ${ }^{19}$

A phase 2, open-label, multicenter noncomparative single-arm trial involved 233 patients taking bedaquiline for 24 weeks, and they were followed up for a further 96 weeks with a clinical visit every 12 weeks. Efficacy was determined by triplicate spot sputum samples during every visit by liquid culture. The drug sensitivity test for bedaquiline and other antitubercular drugs was determined at the baseline and after 24 weeks of bedaquiline administration. At the end of 120 weeks, culture conversion was $73.1 \%$ in MDR-TB, $70.5 \%$ in pre-XDR-TB, and $62.2 \%$ in XDR-TB patients. ${ }^{20}$

Similarly, the study was conducted in Japan to evaluate the safety, efficacy, and pharmacokinetics of bedaquiline in adult Japanese patients who received bedaquiline for 24 weeks or more (maximum 48 weeks) with background regimen (BR). The time required for culture conversion was 14-15 days. But adverse effects, such as abnormal hepatic function, hypoesthesia, nasopharyngitis, acne, and nausea were reported. ${ }^{21}$

To assess long-term outcomes and safety of prolonged bedaquiline treatment for MDR-TB a retrospective cohort study was conducted in 45 patients with MDR-TB. Bedaquiline was administered for a prolonged duration (>190 days) in which 36 patients had shown the favorable outcome, three patients died, five patients were lost to follow-up, and one patient failed and acquired bedaquiline resistance. Several serious adverse effects like QT prolongation occurred but no recurrence was reported. But overall no significant difference in adverse events and outcome was observed when bedaquiline was administered for a prolonged period. Since bedaquiline shows a satisfactory outcome in patients, it was considered that prolonged treatment with bedaquiline was overall safe and also well tolerated. ${ }^{22}$

In another retrospective observational study, it was proved that bedaquiline and surgery can be safely combined in selected patients with specific indications. ${ }^{23} \mathrm{~A}$ higher dose of bedaquiline for long-term does not lead to cardiotoxic or hepatotoxic effects. ${ }^{24}$

In MDR-TB burden countries, the study was conducted to determine the impact of the total cost of adding bedaquiline to $B R$ which showed that by adding bedaquiline to BR the outcome of the patient improved at the same time decreasing the healthcare cost. ${ }^{25}$ Bedaquiline use in patients with MDR-TB and end-stage renal disease was also found to be satisfactory. ${ }^{26}$

Bedaquiline is available as a $100 \mathrm{mg}$ tablet. Bioavailability of the drug increases when it is taken along with food. Once administered, more than $99 \%$ bind to plasma protein. The terminal half-life of the drug is $4-5$ months. The drug undergoes oxidative metabolism by the cytochrome 3A4 enzyme. The metabolite obtained is $N$-monodesmethyl metabolite. This metabolite potency is 4-6 times less when compared with the parent drug. As the drug is metabolized by cytochrome P450 enzyme, when the enzyme inducers or enzyme inhibitors are taken along with bedaquiline, the efficacy of bedaquiline is altered. Elimination is mainly by feces. ${ }^{27}$ The most important side effect is QT prolongation and hepatotoxicity. ${ }^{28}$ Recommended dosage is $400 \mathrm{mg}$ orally daily for 2 weeks followed by $200 \mathrm{mg}$ three times weekly. ${ }^{29}$
The standard drug for MDR-TB has many cons, such as high cost, more drug interactions, and less efficacy but bedaquiline has the ability to overcome these disadvantages and also decreases the time necessary for sputum conversion ${ }^{30}$ and thus provides satisfactory outcome in this form of TB. Though rifampicin interacts with bedaquiline and reduces its bioavailability by $50 \%$, the addition of bedaquiline to the standard drugs, such as pyrazinamide, rifampicin, and isoniazid were found to be effective. ${ }^{31}$ Bedaquiline has bactericidal activity on both dormant, as well as replicating bacteria and thus shortens the duration of TB treatment. ${ }^{32}$ It was also found to be effective in the treatment of leprosy. ${ }^{33}$ Currently, the routine use of bedaquiline for more than 6 months was not recommended by WHO. ${ }^{34}$ But WHO acknowledges that if the regimen is unlikely to achieve cure or if there is the risk of additional drug resistance, then clinicians and national TB programs may be compelled to use bedaquiline beyond 24 weeks in selected MDR-TB patients. ${ }^{35}$

\section{References}

1. Corbett EL, Watt CJ, Walker N, Maher D, Williams BG, Raviglione MC, et al. The growing burden of tuberculosis: global trends and interactions with the HIV epidemic. Arch Intern Med 2003;163(9): 1009-1021. DOI: 10.1001/archinte.163.9.1009.

2. Young DB, Perkins MD, Duncan K, Barry 3rd CE. Confronting the scientific obstacles to global control of tuberculosis. J Clin Invest 2008;118(4):1255-1265. DOI: 10.1172/JCI34614.

3. Sandhu GK. Tuberculosis: current situation, challenges and overview of its control programs in India. J Glob Infect Dis 2011;3(2):143-150. DOI: 10.4103/0974-777X.81691.

4. Nagabushan $\mathrm{H}$, Roopadevi HS. Bedaquiline: a novel antitubercular drug for multidrug-resistant tuberculosis. J Postgrad Med 2014;60(3):300-302. DOI: 10.4103/0022-3859.138772.

5. WHO. Global Tuberculosis Report 2014, vol. 5 Geneva: World Health Organisation; 2014; 54 p. 54.

6. Deoghare S. Bedaquiline: a new drug approved for treatment of multidrug resistant tuberculosis. Indian J Pharmacol 2013;45(5): 536-537. DOI: 10.4103/0253-7613.117765.

7. Lienhardt C, Nahid P, Rich ML, Bansbach C, Kendall EA, Churchyard $\mathrm{G}$, et al. Target regimen profiles for treatment of tuberculosis: a WHO document. Eur Respir J 2017;49(1):1602352. DOI: 10.1183/13993003.02352-2016.

8. Gualano G, Capone S, Matteelli A, Palmieri F. New antituberculosis drugs: from clinical trial to programmatic use. Infect Dis Rep 2016;8(2):6569. DOI: 10.4081/idr.2016.6569.

9. Yadav S, Rawal G, Baxi M. Bedaquiline: a novel antitubercular agent for the treatment of multidrug resistant tuberculosis. J Clin Diagn Res 2016;10(8):FM01-FM02. DOI: 10.7860/JCDR/2016/19052.8286.

10. Kakkar AK, Dahiya N. Bedaquiline for the treatment of resistant tuberculosis: promises and pitfalls. Tuberculosis (Edinb) 2014;94(4):357-362. DOI: 10.1016/j.tube.2014.04.001.

11. Singh A, Prakash V, Kant S, Kumar R, Bhatia A, Verma AK, et al. Bedaquiline: a new hope in treatment of tuberculosis. Int J Health Allied Sci 2016;5(3):172-173. DOI: 10.4103/2278-344X.187830.

12. Lenaerts AJ, Hoff D, Aly S, Ehlers S, Andries K, Cantarero L, et al. Location of persisting mycobacteria in a guinea pig model of tuberculosis revealed by r207910. Antimicrob Agents Chemother 2007;51(9):3338-3345. DOI: 10.1128/AAC.00276-07.

13. Veziris N, Ibrahim M, Louis N, Chauffour A, Truffot-Pernot C, Andries $\mathrm{K}$, et al. A once weekly R207910-containing regimen exceeds activity of the standard daily regimen in murine tuberculosis. Am J Respir Crit Care Med 2009;179(1):75-79. DOI: 10.1164/rccm.200711-17360C.

14. Ibrahim M, Andries K, Lounis N, Chauffour A, Truffot-Pernot C, Jarlier V, et al. Synergistic activity of R207910 combined with pyrazinamide against murine tuberculosis. Antimicrob Agents Chemother 2007;51(3):1011-1015. DOI: 10.1128/AAC.00898-06. 
15. Zhang $Y$, Wade MM, Scorpio A, Zhang $H$, Sun Z. Mode of action of pyrazinamide: disruption of Mycobacterium tuberculosis membrane transport and energetics by pyrazinoic acid. J Antimicrob Chemother 2003;52(5):790-795. DOI: 10.1093/jac/dkg446.

16. Diacon AH, Pym A, Grobusch M, Patientia R, Rustomjee R, PageShipp L, et al. The diarylquinoline TMC207 for multidrug-resistant tuberculosis. N Engl J Med 2009;360(23):2397-2405. DOI: 10.1056/ NEJMoa0808427.

17. Diacon AH, Pym A, Grobusch M, de los Rios JM, Gotuzzo E, Vasilyeva I, et al. Multidrug resistant tuberculosis and culture conversion with bedaquiline. N Engl J Med 2014;371(8):723-732. DOI: 10.1056/ NEJMoa1313865.

18. Guglielmetti L, Le Du D, Jachym M, Henry B, Martin D, Caume E, et al. Compassionate use of bedaquiline for the treatment of multidrug resistant and extensively drug-resistant tuberculosis: interim analysis of a French cohort. Clin Infect Dis 2015;60(2):188-194. DOI: 10.1093/ $\mathrm{cid} / \mathrm{ciu} 786$

19. Ndjeka N, Conradie F, Schnippel K, Hughes J, Bantubani N, Ferreira H, et al. Treatment of drug-resistant tuberculosis with bedaquiline in a high HIV prevalence setting: an interim cohort analysis. Int J Tuberc Lung Dis 2015;19(8):979-985. DOI: 10.5588/ijtld.14.0944.

20. Pym AS, Diacon H, Tang S-J, Francesca C, Manfred D, Charoen C, et al. Bedaquiline in the treatment of multidrug and extensively drug resistant tuberculosis. Eur Respir J 2016;47(2):564-574. DOI: 10.1183/13993003.00724-2015.

21. Tsuyuguchi K, Sasaki Y, Mitarai S, Kurosawa K, Saito Y, Koh T. Safety, efficacy, and pharmacokinetics of bedaquiline in Japanese patients with pulmonary multidrug resistant tuberculosis: an interim analysis of an open-label, phase 2 study. Respir Investig 2019;57(4):345-353. DOI: 10.1016/j.resinv.2019.01.001.

22. Guglielmetti L, Jaspard M, Le Dû D, Marie L, Dhiba M-O, Christine $B$, et al. Long-term outcome and safety of prolonged bedaquiline treatment for multidrug-resistant tuberculosis. Eur Respir J 2017;49(3):1601799. DOI: 10.1183/13993003.01799-2016.

23. Borisoy SE, D'Ambrosio L, Centis R, Tiberi S, Dheda K, Alffenaar $J W$, et al. Outcomes of patients with drug resistant tuberculosis treated with bedaquiline-containing regimens and undergoing adjunctive surgery. J Infect 2019;78(1):35-39. DOI: 10.1016/j.jinf.2018. 08.003
24. Telnov O, Alvarez V, Graglia E, Molfino L, Cros PD, Rich M. Bedaquiline overdose: a case report. Int J Infect Dis 2019;84:138-140. DOI: 10.1016/j.ijid.2019.03.035.

25. Lu X, Smare C, Kambili C, Khoury ACE, Wolfson LJ. Health outcomes of bedaquiline in the treatment of multidrug resistant tuberculosis in selected high burden countries. BMC Health Serv Res 2017;17(1):87. DOI: 10.1186/s12913-016-1931-3.

26. Park S, Lee KM, Kim I, Mok J. The use of bedaquiline to treat patients with multidrug-resistant tuberculosis and end stage renal disease: a case report. Indian J Infect Dis 2018;76:88-90. DOI: 10.1016/ j.ijid.2018.09.009.

27. U.S. Food and Drug Administration. SIRTURO Prescribing Information 2013. pp. 1-25.

28. Fox GJ, Menzies D. A review of the evidence for using bedaquiline (TMC207) to treat multidrug resistant tuberculosis. Infect Dis Ther 2013;2(2):123-144. DOI: 10.1007/s40121-013-0009-3.

29. Matteelli A, Carvalho ACC, Dooley KE, Kritski A. TMC207: the first compound of a new class of potent anti-tuberculosis drugs. Future Microbiol 2010;5(6):849-858. DOI: 10.2217/fmb.10.50.

30. Field SK. Bedaquiline for the treatment of multidrug-resistant tuberculosis: great promise or disappointment? Ther Adv Chronic Dis 2015;6(4):170-184. DOI: 10.1177/2040622315582325.

31. Lounis N, Gevers T, Van Den Berg J, Andries K. Impact of the interaction of R207910-with rifampin on the treatment of tuberculosis studied in the mouse model. Antimicrob Agents Chemother 2008;52(10): 3568-3572. DOI: 10.1128/AAC.00566-08.

32. Koul A, Vranckx L, Dendouga N, Balemans W, Van den Wyngaert I, Vergauwen K, et al. Diarylquinolines are bactericidal for dormant mycobacteria as a result of disturbed ATP homeostasis. J Biol Chem 2008;283(37):25273-25280. DOI: 10.1074/jbc.M803899200.

33. Gelber R, Andries K, Paredes RM, Andaya CE, Burgos J. The diarylquinolone R207910 is bactericidal against Mycobacterium lepraein mice at low dose and administered intermittently. Antimicrob Agents Chemother 2009;53(9):3989-3991. DOI: 10.1128/AAC.00722-09.

34. World Health Organisation. WHO treatment guidelines for multi drug and rifampicin resistant tuberculosis 2018 update.

35. World Health Organisation. WHO best practice statement on the off label use of Bedaquiline and Delamanid for the treatment of multidrug resistant tuberculosis. 2017. 